\title{
Support Information: Non-Equilibrium Dynamics of Vesicles and Micelles by Self-Assembly of Block Copolymers with Double Thermoresponsivity
}

\author{
Yu-Hang Tang ${ }^{* 1}$, Zhen $\mathrm{Li}^{1}$, Xuejin $\mathrm{Li}^{1}$, Mingge Deng ${ }^{1}$, and George Em Karniadakis ${ }^{\dagger 1,2}$ \\ ${ }^{1}$ Division of Applied Mathematics, Brown University, Providence, Rhode Island 02912, USA \\ ${ }^{2}$ Collaboratory on Mathematics for Mesoscopic Modeling of Materials, Pacific Northwest National \\ Laboratory, Richland, Washington 99354, USA
}

\section{Details of Simulation}

\subsection{Thermoresponsive Micelles}

DPD systems of size $179.4 \mathrm{~nm} \times 179.4 \mathrm{~nm} \times 179.4 \mathrm{~nm}$, or $60 r_{c} \times 60 r_{c} \times 60 r_{c}$, were initialized by generating linear $A_{20} B_{20}$ polymers using a random walk algorithm. Each system contains 43,200 polymer beads and 820,800 solvent particles, corresponding to a polymer volume fraction of $5 \%$. A boundary condition that emulates the effect of a semipermeable membrane was applied in all three dimensions: the boundaries are periodic for solvent particles but impenetrable for polymers. An integral conservative force as described in Ref [1] is used to prevent polymers from approaching the boundary while introducing minimal perturbation to particle density near the boundaries.

The parameters in Table 1 were used for simulating different scenarios of thermally induced self assembly. The simulations were started by first keeping the system temperature at $282 \mathrm{~K}$ for $15000 \tau$, or $3.75 \mathrm{~ms}$. The temperature was then linearly elevated to $318 \mathrm{~K}$ in $3.75 \mathrm{~ms}$ and then further maintained for another $3.75 \mathrm{~ms}$.

To heat up or cool down the eDPD system, each eDPD particle is coupled with a thermal background of desired temperature $T^{B}(t)$. This induces a heat flux

$$
Q_{i}^{B}(t)=\lambda C_{\mathrm{v}}\left[T^{B}(t)-T_{i}(t)\right],
$$

where $\lambda$ is a relaxation factor chosen to be $5 \times 10^{-5}$ in all our simulations. It is shown in Fig. 1 that the method can effectively control the system temperature while still preserving a natural (Gaussian) particle temperature distribution.

The effect of heating rate on the micelle inversion mechanism was examined by repeating the simulation for case $A$, but eliminating the linear heating phase of $3.75 \mathrm{~ms}$. Instead, the system temperature was raised almost instantly after $t_{1}$.

We further studied the effect of $\Delta \theta \doteq \theta^{\mathrm{U}}-\theta^{\mathrm{L}}$ on the micellar inversion pathway. This is done by simulating case $A$ from the same initial configuration but varying $\theta^{\mathrm{U}}$ and $\theta^{\mathrm{L}}$ of the blocks while keeping $\left(\theta^{\mathrm{U}}+\theta^{\mathrm{L}}\right) / 2 \equiv 300 K$.

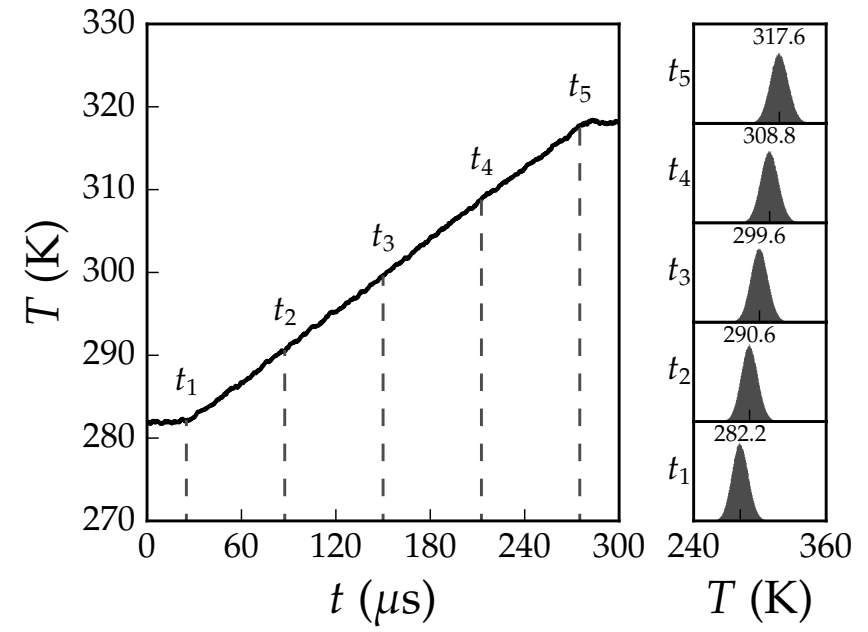

Supplementary Figure 1: Temperature evolution and distribution during a heating process as controlled by a coupled thermal background.

\subsection{Thermoresponsive Vesicles}

To simulate thermoresponsive vesicles and membranes we used an $\mathrm{LCST}_{2} \mathrm{~N}_{5} U C S T_{2}$ triblock copolymer, whose conservative force constants within itself and between water are given in Table 1. The polymer was chosen based on two criteria: 1) linear symmetry is necessary to ensure that the inverted membrane is structurally similar to the one before inversion; 2) shorter chains are preferred in order to contain an entire vesicle within the finite spatial and temporal extent accessible by our simulation.

Unilamellar vesicles (ULVs) were constructed by randomly inserting 6511 polymers into a spherical shell of outer diameter $112 \mathrm{~nm}$ and thickness $7.48 \mathrm{~nm}$, The spherical shell was placed at the center of a simulation box of $179.4 \mathrm{~nm} \times 179.4 \mathrm{~nm} \times 179.4 \mathrm{~nm}$, or $60 r_{c} \times 60 r_{c} \times 60 r_{c}$. Solvent particles were then inserted into the box to obtain an overall number density of 4 . Each system was then equilibrated by an isothermal pre-run of $10000 \tau$, or $2.5 \mathrm{~ms}$. 
Table 1: Summary of the conservative force parameters used throughout the study. In all equations $T_{i j}=\left(T_{i}+T_{j}\right) / 2$ and $T_{i} \doteq T_{i}^{*} / T_{0}$ is the reduced temperature of particle $i$.

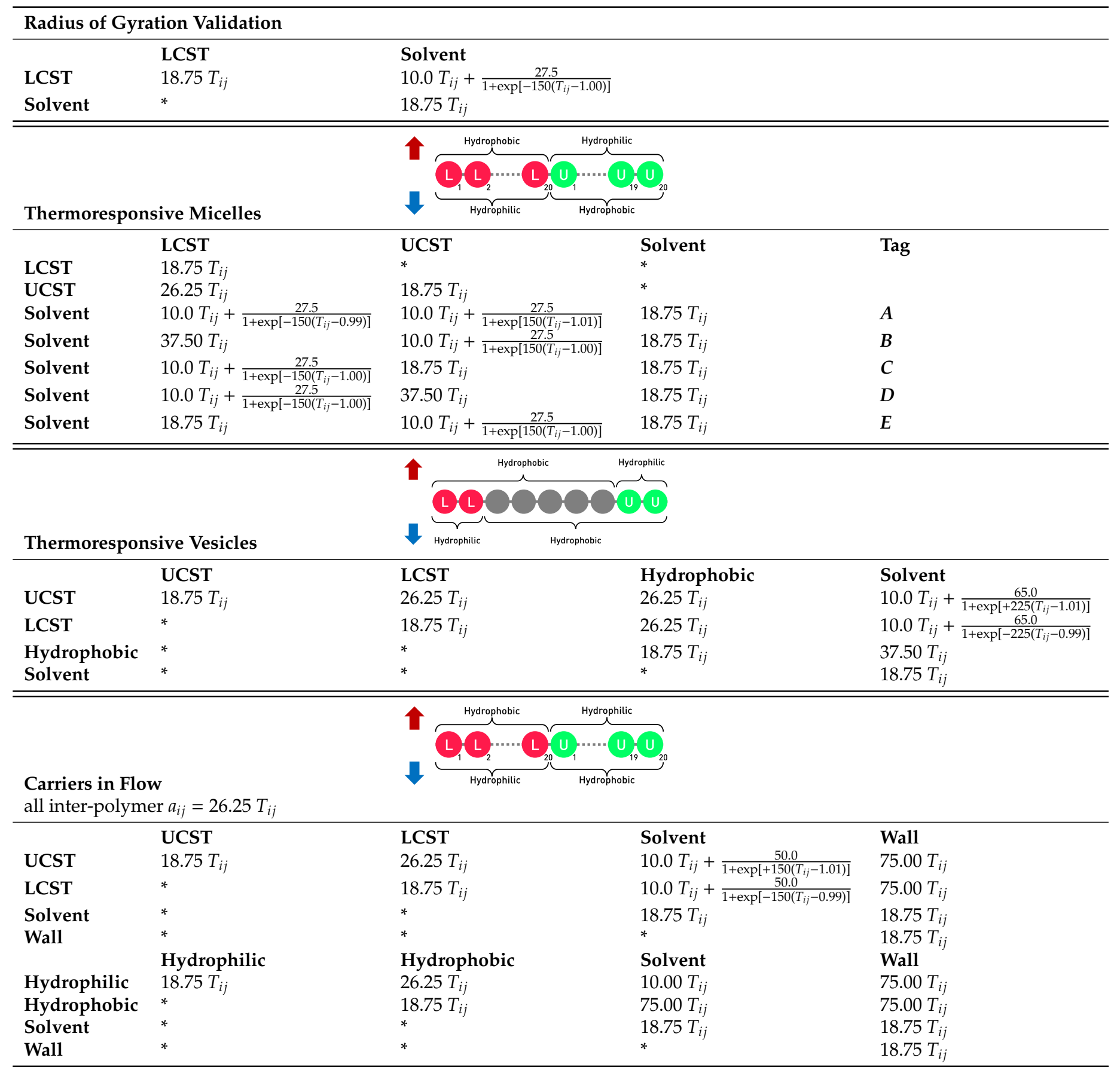

To quantify the collapse and subsequent release of the containing fluid of vesicles, a thermal loading test was performed where ULVs were subjected to repeated heating-cooling cycles. Let $\phi$ be the duration of each cycle, $f \doteq 1 / \phi$ the loading frequency, and $T_{h i}, T_{l o}$ the highest and lowest temperature, the temperature of the system is controlled as a function of time $t$ :

$$
T(t)=\left\{\begin{array}{lr}
\mathrm{L}\left(T_{l o}, T_{h i}, t, 0\right), & 0 \leq t<\frac{1}{4} \phi \\
T_{h i}, & \frac{1}{4} \phi \leq t<\frac{1}{2} \phi \\
\mathrm{L}\left(T_{h i}, T_{l o}, t, \frac{1}{2} \phi\right), & \frac{1}{2} \phi \leq t<\frac{3}{4} \phi \\
T_{l o}, & \frac{3}{4} \phi \leq t<\phi
\end{array}\right.
$$

where $L\left(T_{0}, T_{1}, t, t_{0}\right) \doteq T_{0}+\left(T_{1}-T_{0}\right) \cdot\left(t-t_{0}\right) / \frac{\phi}{4}$ is essentially a linear interpolation from $\left(t_{0}, T_{0}\right)$ to $\left(t_{0}+\frac{\phi}{4}, T_{1}\right)$. 
We tested 8 frequencies corresponding to $\phi$ ranging between $5 \mu \mathrm{s}$ and $1.25 \mathrm{~ms}$ and simulated 72 ensembles for each of the frequency, giving rise to a total of 576 DPD systems each containing 864000 particles simulated for $\sim 22 \mathrm{M}$ time steps, or $\sim 55 \mathrm{~ms}$ in physical time. The computation was done on the Titan supercomputer at Oak Ridge Leadership Computing Facility using 18432 nodes for 24 hours. The time of observed collapse events of the vesicles was used to obtain a maximum likelihood estimate of a right-truncated gamma distribution:

$$
p(t ; a, b)=\frac{t^{a-1}}{\Gamma(a) b^{a}} e^{-\frac{t}{b}}
$$

with which half-life of the vesicles is then calculated from. However, for intermediate frequencies many vesicles can survive for a long time and the available information appeared insufficient for getting a reliable estimate of a long-tail distribution. In this case only the final survival percentage is given.

\subsection{Molecular Mechanism of Inversion}

To extract patterns from thousands of noisy molecular trajectories, the Proper Orthogonal Decomposition (POD) method was employed [2]. We assembled a N-by-M matrix $A$, where each row of A represents one molecular trajectory over time. A singular value decomposition of $A$ gives

$$
A_{N \times M}=U_{N \times N} \Sigma_{N \times M} V_{M \times M}^{T}
$$

where columns of $V$ represent the orthogonal modes, arranged in decreasing magnitude of importance, decomposed from the inversion dynamics. The diagonal elements of $\Sigma$, i.e. the singular values of $A$, quantify the importance of each mode.

\subsubsection{Micelle}

To reveal the molecular movement patterns of thermoresponsive micelles, we construct periodic simulation boxes of size $59.8 \mathrm{~nm} \times 59.8 \mathrm{~nm} \times 59.8 \mathrm{~nm}$, or $20 r_{c} \times 20 r_{c} \times 20 r_{c}$, each containing $\sim 100 \mathrm{LCST}_{8} U \mathrm{UCST} T_{8}$ polymers placed near the center of the box. Solvent particles were then filled to achieve an overall number density of 4 . The system was equilibrated at $T=291 \mathrm{~K}$ for $100 \tau$ before an instant temperature jump to $309 \mathrm{~K}$ was applied to trigger inversion. The radial component of the center of mass (COM) trajectories of the LCST and UCST block of each individual molecule was collected for POD analysis. We further aggregate data obtained from 100 independent ensembles to improve result accuracy and smoothness. The matrix $A$ in this case is 10436-by-720:

$$
A=\left(\begin{array}{ccccccc}
l_{0}^{1} & l_{10}^{1} & \cdots & l_{3600}^{1} & u_{0}^{1} & \cdots & u_{3600}^{1} \\
l_{0}^{2} & l_{10}^{2} & \cdots & l_{3600}^{2} & u_{0}^{2} & \cdots & u_{3600}^{2} \\
\vdots & \vdots & \ddots & \vdots & \vdots & \ddots & \vdots \\
l_{0}^{10436} & l_{10}^{10436} & \cdots & l_{3600}^{10436} & u_{0}^{10436} & \cdots & u_{3600}^{10436}
\end{array}\right)
$$

where $l_{j}^{i}$ and $u_{j}^{i}$ are the radial component of the center of mass (COM) of the LCST and UCST blocks of molecule $i$ with respect to the center of mass of the entire micelle at time $j \cdot \delta t \cdot \tau$ after the onset of inversion.

Fig. 2A illustrated the morphology of the micelle before and after the inversion. A single dominant mode, which corresponds to local inversion, can be seen in Fig. 2B.
A

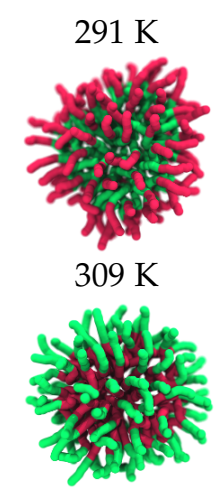

LCST
B POD modes

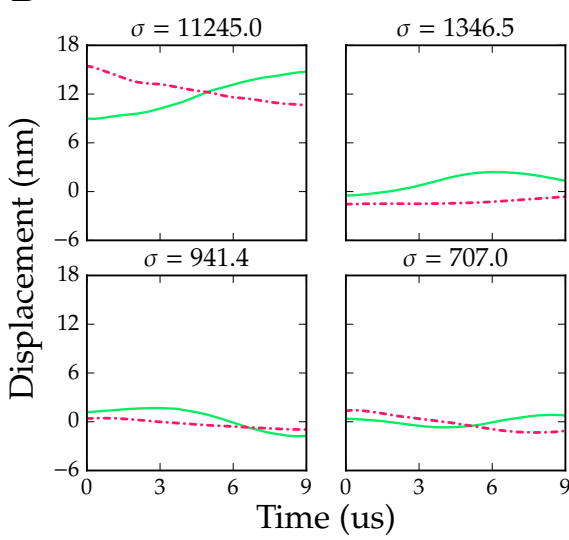

UCST
Supplementary Figure 2: Micelles formed by a $L_{8} U_{8}$ thermoresponsive block copolymer invert upon instantaneous heating though local inversion of individual molecules. (A) an example of the micelle before and after inversion; (B) POD modes of UCST and LCST block trajectory during inversion; LCST, UCST blocks are colored in red dashed and green solid lines, respectively.

\subsubsection{Membrane}

To identify movement patterns of individual molecules during the inversion process, a two-dimensional periodic bilayer membranes was used as a zoomed-in model of the vesicle surface. The membrane was initialized by randomly placing 6784 polymer molecules within a xz-parallel slab measuring $11.96 \mathrm{~nm}$ thick in a periodic box of $179.4 \mathrm{~nm} \times 179.4 \mathrm{~nm} \times$ $179.4 \mathrm{~nm}$, or $60 r_{c} \times 60 r_{c} \times 60 r_{c}$, while the rest of the system is filled with solvent particles for an overall number density of 4. The system was first equilibrated at $291 \mathrm{~K}$ for $2.5 \mathrm{~ms}$, or $10000 \tau$. After that, the system temperature was elevated to $309 \mathrm{~K}$ almost instantaneously to trigger the inversion. The matrix $A$ in this case is $6784-b y-720$ :

$$
A=\left(\begin{array}{ccccccc}
l_{0}^{1} & l_{10}^{1} & \cdots & l_{3600}^{1} & u_{0}^{1} & \cdots & u_{3600}^{1} \\
l_{0}^{2} & l_{10}^{2} & \cdots & l_{3600}^{2} & u_{0}^{2} & \cdots & u_{3600}^{2} \\
\vdots & \vdots & \ddots & \vdots & \vdots & \ddots & \vdots \\
l_{0}^{6874} & l_{10}^{6874} & \cdots & l_{3600}^{6874} & u_{0}^{6874} & \cdots & u_{3600}^{6874}
\end{array}\right)
$$

where $l_{j}^{i}$ and $u_{j}^{i}$ are the $y$ component of the center of mass (COM) of the LCST and UCST blocks of molecule $i$ at time $j \cdot \delta t \cdot \tau$ after the onset of inversion. In other words, the trajectory of the LCST and UCST blocks of each molecule was 
sampled at a frequency of $4 \mathrm{ps}^{-1}$ and used as one record, while POD was used to discover the patterns across the records.

\subsection{Thermoresponsive Carrier in Flow}

The periodic cylindrical tube measures $800 \mathrm{~nm}\left(267.56 r_{c}\right)$ long with a radius of $59.8 \mathrm{~nm}\left(20 r_{c}\right)$. The temperature at the first and second half of the channel wall along the axial direction was kept at $288 \mathrm{~K}$ and $312 \mathrm{~K}$, respectively. The no-slip boundary condition and a Dirichlet boundary condition for temperature were applied using an integral method as described in Ref. [3]. An aggregate, either micelle or vesicle, is centered on the axis, $120 \mathrm{~nm}$ from the left end of the tube. In the case of micelles, a sphere of radius $21 \mathrm{~nm}$ was randomly filled with $\sim 210 A_{20} B_{20}$ polymers. Three scenarios were simulated, which correspond to micelles formed by a LCST-hydrophilic copolymer (LCST ${ }_{20}$ Hydrophilic $_{20}$ ), a LCST-UCST copolymer $\left(\mathrm{LCST}_{20} \mathrm{UCST}_{20}\right)$, and a non-responsive amphiphilic copolymer (Hydrophilic ${ }_{20}$ Hydrophobic $_{20}$ ), respectively, using parameters given in Table 1 . In the case of vesicles, a hollow spherical shell of outer radius $35.88 \mathrm{~nm}$ and thickness 11.96 $\mathrm{nm}$ was randomly filled with $\sim 2700 \mathrm{LCST}_{2} \mathrm{~N}_{5} U C S T_{2}$ polymers. Solvent particles were then inserted to achieve an overall particle number density of 4 . A uniform pressure gradient was applied in the positive $x$ direction to drive the flow for a terminal velocity of $2 \mathrm{~mm} / \mathrm{s}$. The simulation was performed for $50000 \tau$, or $12.5 \mathrm{~ms}$ for micellar systems and $40000 \tau$, or $10 \mathrm{~ms}$ for vesicle systems. Ensemble averaging was used to obtain smooth statistics of mean temperature, horizontal velocity, and interface interaction energy along the flow direction. The disintegration process of the LCST-hydrophilic copolymer micelle is shown in Fig. 3, while more discussion can be found in the main text.

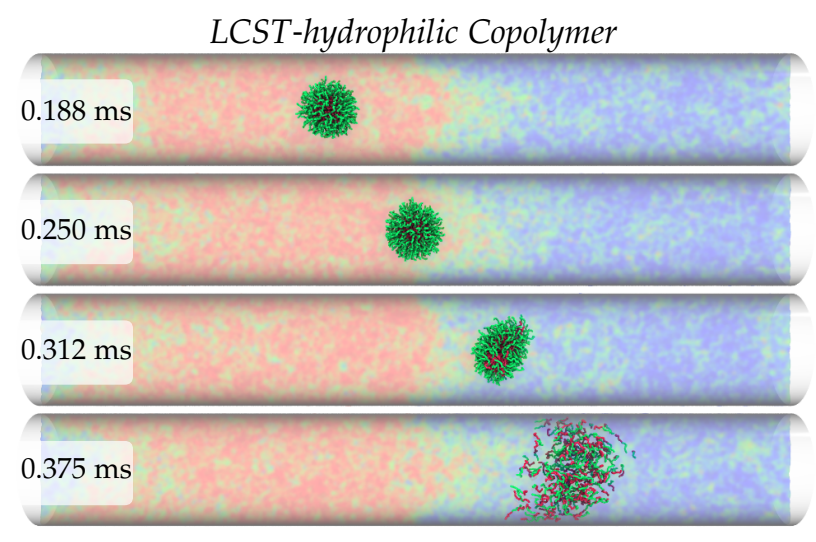

Supplementary Figure 3: A LCST-hydrophilic copolymer micelle disintegrated after crossing the thermal interface and entering a high-temperature zone where the LCST blocks became hydrophilic.

\section{Acknowledgment}

This work was supported by the Department of Energy (DOE) Collaboratory on Mathematics for Mesoscopic Modeling of Materials (CM4). Simulations were carried out at the Oak Ridge Leadership Computing Facility through the Innovative and Novel Computational Impact on Theory and Experiment program at Oak Ridge National Laboratory under project BIP102 and BIP118. Particle simulation results were visualized with VMD [4]. YHT thanks Changho Kim for helpful discussions on polymer diffusivity. YHT acknowledges partial financial support from an IBM Ph.D. Scholarship Award.

\section{References}

[1] Zhen Li, Yu-Hang Tang, Huan Lei, Bruce Caswell, and George Em Karniadakis. Energy-conserving dissipative particle dynamics with temperature-dependent properties. Journal of Computational Physics, 265(0):113 - 127, 2014.

[2] Anindya Chatterjee. An introduction to the proper orthogonal decomposition. Current science, 78(7):808-817, 2000.

[3] Zhen Li, Yu-Hang Tang, Huan Lei, Bruce Caswell, and George Em Karniadakis. Energy-conserving dissipative particle dynamics with temperature-dependent properties. Journal of Computational Physics, 265:113-127, 2014.

[4] William Humphrey, Andrew Dalke, and Klaus Schulten. VMD - Visual Molecular Dynamics. Journal of Molecular Graphics, 14:33-38, 1996. 